\title{
Pattern of pulmonary venous blood flow in the hypoplastic left heart syndrome in the fetus
}

\author{
D J Better, H D Apfel, V Zidere, L D Allan
}

Department of Pediatric Cardiology, Babies Hospital, Columbia Presbyterian Medical Center, 3959 Broadway, New York, NY 10032, USA

D J Better

H D Apfel

V Zidere

L D Allan

Correspondence to:

Dr Allan.

email:1a48@columbia.edu

Accepted for publication 6 January 1999

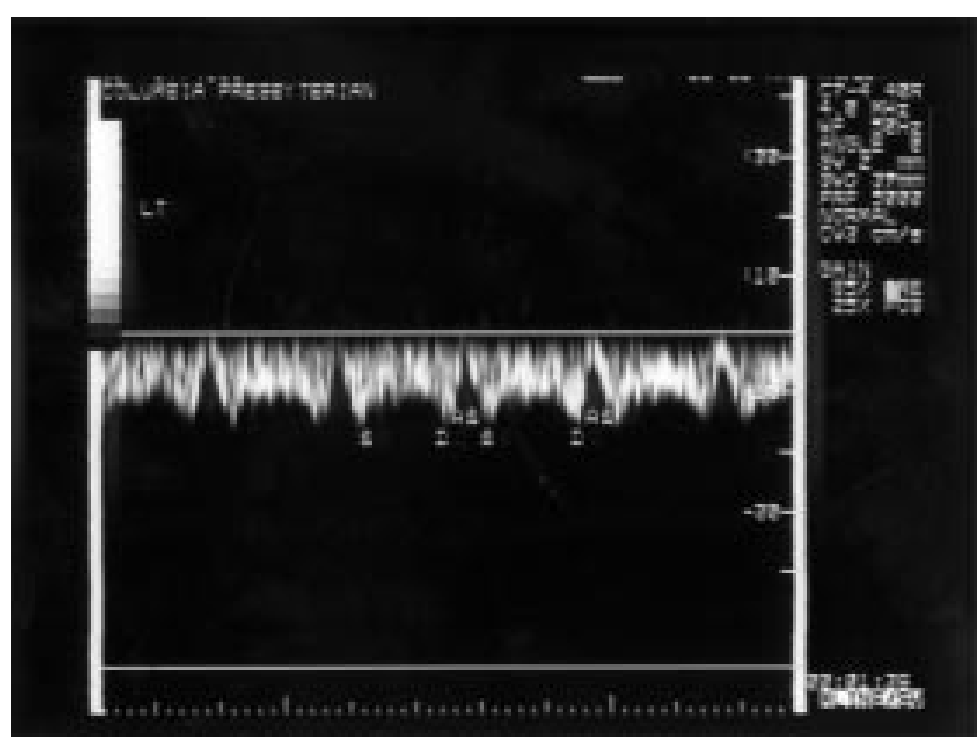

Figure 1 The normal pattern of pulmonary vein flow in a fetus. There is a systolic peak (S) followed by an early diastolic peak (D) and flow cessation or reversal during late diastole or atrial systole (AS). The systolic and diastolic velocities are similar and there is a mild rise in velocity of forward flow with advancing gestation with a range of $10 \mathrm{~cm} / \mathrm{s}$ to 40 $\mathrm{cm} / \mathrm{s}$ between 16 weeks and term. tained in 18 fetuses with left atrial outflow atresia and compared with 77 controls, adjusted for gestational age. Postnatal echocardiograms were available for analysis in 13 of 18 neonates.

Setting-A tertiary referral centre for fetal cardiology and paediatric cardiac surgery.

Results-Fetuses with hypoplastic left heart syndrome were different from controls in all pulmonary vein indices measured. As assessed from the postnatal echocardiogram, there were seven fetuses with a restrictive atrial septum. In these fetuses, the systolic flow velocity

\begin{abstract}
Objective-To determine whether restriction at the atrial septum in the newborn with hypoplastic left heart syndrome can be predicted accurately by examining the pattern of pulmonary venous flow in the fetus. A restrictive atrial septum can contribute to haemodynamic instability before surgery for this lesion and has been associated with an increased mortality.

Design-Pulmonary venous pulsed Doppler tracings were compared between
\end{abstract} fetuses with hypoplastic left heart syndrome and controls. The size of the atrial septal defect on the postnatal echocardiogram was graded according to the degree of restriction. Pulsed Doppler tracings of pulmonary venous blood flow were ob-

(2) important influence on the results. A restrictive atrial septum has been suggested as a risk factor for Norwood palliation. ${ }^{4}$ The ability to predict restriction of the atrial defect prenatally may allow earlier surgery to be planned. However, in our experience direct evaluation of the atrial septum in the fetus in the setting of mitral atresia has proved difficult. It is usually easy to see if the foramen is widely patent but conversely, a normally sized defect can be difficult to distinguish from a restrictive defect. This may be because the atrial communication in this setting is often high and posterior in position. In addition, visualisation of the defect requires good colour penetration and a perpendicular orientation to the atrial defect, which is not always achievable, especially in late pregnancy. For this reason, we have focused on the pulsed Doppler pattern of pulmonary vein flow, which is technically always readily obtainable.

Pulmonary venous flow patterns are related to left atrial haemodynamics and have been studied intensively, particularly in the adult. ${ }^{6-8}$ The normal pattern of pulmonary venous blood flow has been described previously in the 
Table 1 Comparison of pulmonary vein velocities in the three groups of patients evaluated

\begin{tabular}{lllll}
\hline Atrial septum & $\begin{array}{l}\text { Peak systolic } V \\
\text { mean }(\mathrm{cm} / \mathrm{s})\end{array}$ & $\begin{array}{l}\text { Peak diastolic } V \\
\text { mean }(\mathrm{cm} / \mathrm{s})\end{array}$ & $\begin{array}{l}\text { S/D ratio } \\
\text { mean }\end{array}$ & $\begin{array}{l}\text { Peak rev V } \\
\text { mean }(\mathrm{cm} / \mathrm{s})\end{array}$ \\
\hline Normal $(\mathrm{n}=77)$ & 21.4 & 20.0 & 1.1 & 4.0 \\
HLHS (open, $\mathrm{n}=6)$ & 27.7 & 18.9 & $1.5^{\star}$ & $1.4 \dagger$ \\
HLHS (restrictive, $\mathrm{n}=7)$ & $33.7 \dagger$ & 16.1 & $2.26 \dagger$ & $22.0 \dagger$ \\
\hline
\end{tabular}

${ }^{\star} \mathrm{p}<0.05 ; \mathrm{tp}<0.01 v$ control group. Values which achieved statistical significance are shown. HLHS, hypoplastic left heart syndrome; rev, reversal wave; S/D, ratio of systolic to diastolic velocities; $\mathrm{V}$, velocity.

fetus and shows a similar pattern to that found in postnatal life. ${ }^{9}{ }^{10}$ There is forward flow in systole and diastole, and during atrial systole there is cessation of flow or flow reversal at end diastole (fig 1). In this study, we compared the pattern of pulmonary venous blood flow in the fetuses with the hypoplastic left heart syndrome with the normal pattern.

\section{Methods}

Pulsed Doppler tracings of pulmonary venous blood flow were obtained in 77 control fetuses with a structurally normal heart and in 18 fetuses where the outflow from the left atrium was atretic. In 17 of these 18 cases there was mitral and aortic atresia, and in one there was atrioventricular discordance with an absent left connection. The Doppler sample volume was placed in a pulmonary vein at its junction with the left atrium using colour flow mapping to guide positioning. Tracings were obtained parallel to the direction of flow, with the filter at the lowest setting $(50 \mathrm{~Hz})$. The peak systolic, diastolic, and reverse flow velocities, and the systolic to diastolic ratio ( $\mathrm{S} / \mathrm{D}$ ratio), were measured and compared with the values obtained for these variables in the normal controls.

Postnatal echocardiograms were examined retrospectively by an observer unaware of the prenatal findings. In five cases, postnatal studies were unavailable. This was because of termination of pregnancy in three cases and delivery in another centre in two. Studies were available in the remaining 13 cases, obtained

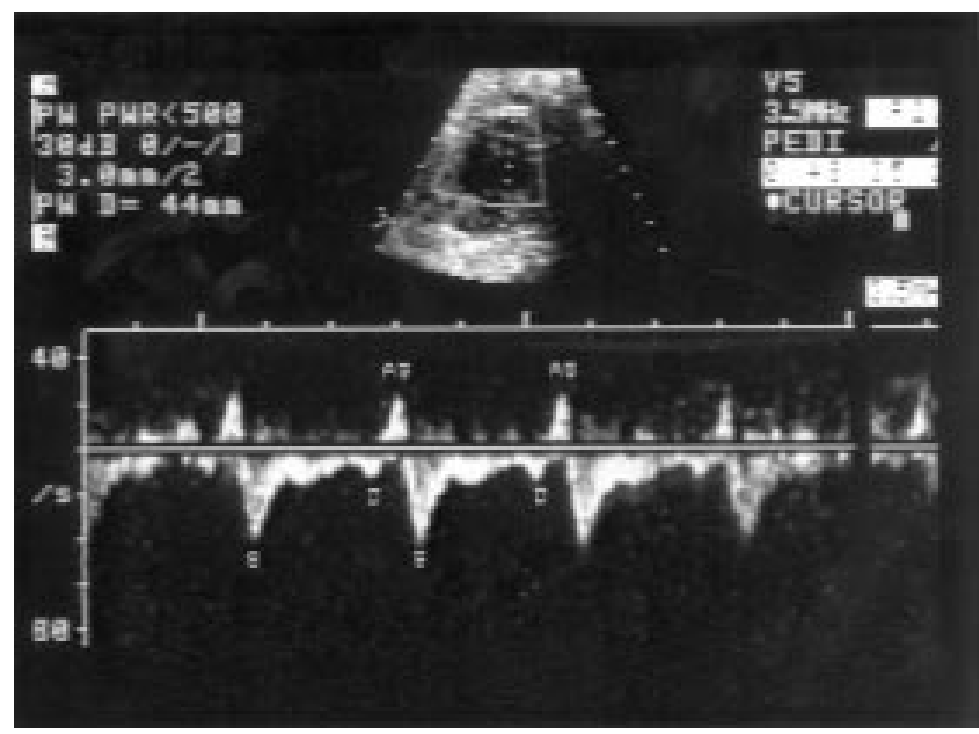

Figure 2 The pattern of pulmonary venous flow in a fetus with the hypoplastic left heart syndrome who had a severely restrictive atrial septum postnatally. The systolic and reverse waves were higher than normal and the diastolic lower than normal for gestational age. The systolic/diastolic ratio was also abnormal.

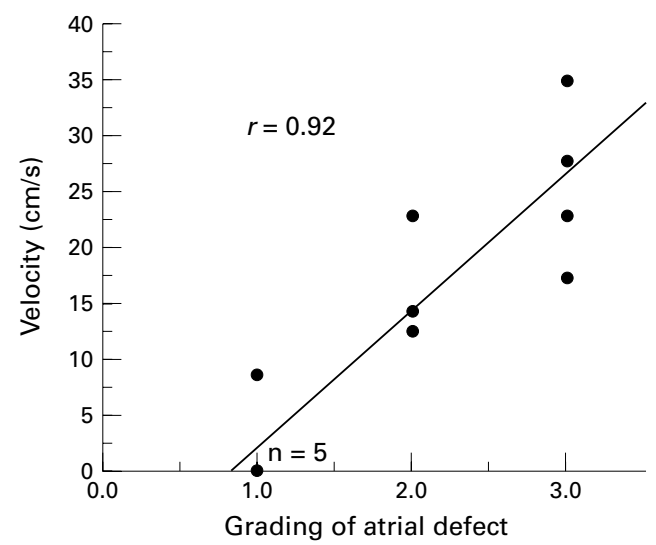

Figure 3 The velocity of the peak reversal wave plotted against the grading of the degree of restriction at the atrial septum on the postnatal echocardiogram, with grade 1 being unrestrictive and grade 3 the most restrictive. Note that there were five fetuses with no flow reversal, represented by a single point on the baseline.

within hours of birth. The size of the atrial defect was graded on a scale of 1 to 3 , with 1 being the least restrictive. The degree of obstruction was determined by a combination of the degree of bulging of the atrial septum to the right, by the size of the defect on cross sectional imaging, and the presence of flow acceleration across the communication on colour flow Doppler interrogation.

STATISTICAL ANALYSIS

The hypoplastic left heart group was compared with controls by an unpaired $t$ test. Where there were more than two groups (restrictive and unrestrictive hypoplastic left heart group and controls), results were compared by analysis of covariance. Covariance was also used to account for the influence of gestational age on the variables studied. Post hoc tests of individual group means were performed using a Bonferroni procedure. The correlation coefficient was calculated using standard methods.

\section{Results}

PULMONARY VENOUS FLOW IN PATIENTS $v$

CONTROLS

There were 77 control studies, gestational age range 16 to 38 weeks, mean of 25.6 weeks. There were 13 fetuses with hypoplastic left heart syndrome, gestational age range 17 to 37 weeks, mean of 28.9 weeks. This difference in gestational age was not statistically significant. In the group of fetuses with the hypoplastic left heart syndrome and a restrictive atrial septum, the systolic velocities differed significantly from the normal range $(\mathrm{p}<0.01)$. There was a mild decrease in diastolic velocity, which did not achieve statistical significance, and a mild increase in the $\mathrm{S} / \mathrm{D}$ ratio above the normal range $(\mathrm{p}<0.05)($ table 1$)$.

FETAL PULMONARY VENOUS FLOW $v$ POSTNATAL FORAMEN OVALE "RESTRICTIVENESS"

In six of 13 postnatal echocardiograms available to us, the atrial defect was graded as widely patent. In seven, it was considered moderately restricted (four cases) and severely restricted (three cases). A postnatally restrictive atrial 
septum correlated $(r=0.92)$ with flow reversal above the normal range on pulmonary venous Doppler flow profile in all seven fetuses (figs 2 and 3).

ANALYSIS OF COVARIANCE

When the pulmonary vein findings in the seven fetuses with a restrictive septum were compared with the normal controls and with those fetuses in the hypoplastic left heart group with an unrestrictive atrial septum, the peak systolic velocity was significantly different from the controls. In addition, the peak reverse velocity and the $\mathrm{S} / \mathrm{D}$ ratio differed both from the controls and from those with an open septum (table 1).

\section{Discussion}

In the human fetus, as much as $20 \%$ of the combined cardiac output passes to the lungs ${ }^{11}$ and returns to the left atrium as pulmonary venous return. If the atrioventricular connection from the left atrium is atretic, the pulmonary venous return must pass to the right atrium through the foramen ovale, reversing the normal direction of flow at this site. The foramen ovale flap (or primum tissue) normally lies in the left atrial cavity, pushed away from the foramen defect by a jet of blood which is derived from the ductus venosus. In mitral atresia, the foramen flap is opposed to the atrial septum, decreasing the size of the foramen defect or even closing it completely; alternatively, the flap valve herniates into the right atrium. In order to examine the atrial septum directly in the fetus, the septum must be imaged with the ultrasound beam perpendicular to it. At this orientation, the colour flow map is optimised for displaying the direction of flow and turbulence, if present. Imaging in this way is often difficult, especially in late pregnancy and particularly in the fetus with the hypoplastic left heart syndrome, when the interatrial communication is often in a high secundum position. The pulmonary vein findings in the hypoplastic left heart group as a whole were different from controls. By analysis of variance, those cases with a restrictive atrial septum were more different from controls than those with an open septum.

The early systolic flow velocity in the pulmonary venous tracing represents the "sucking" effect of atrial relaxation and is higher than normal in the setting of atrioventricular valve atresia. The early diastolic phase of the tracing normally represents forward flow across the mitral valve but in the setting of atrioventricular valve atresia must indicate flow across the foramen ovale. This had a tendency to be less than normal, particularly in the fetuses who proved to have a restrictive septum, but this did not achieve statistical significance. The increased reversal of flow during atrial systole seen in those fetuses who proved to have a restrictive atrial septum postnatally is consistent with the left atrium contracting against not only a closed atrioventricular valve, but also a restrictive "run-off" through the atrial septal defect. The only route for the blood in the left atrium in this circumstance is back into the pulmonary veins. Where there is a restrictive atrial septum, the increase in systolic flow may be caused by a rise in pressure and volume within the pulmonary veins relative to the atrium, owing to the restricted flow during diastole. The fact that a restrictive atrial septum is present from at least the midtrimester in some fetuses (our youngest fetus with this finding was 25 weeks' gestation) is of interest. This may further increase pulmonary resistance and potentially cause vascular damage in this subset of cases of hypoplastic left heart syndrome.

The proportion of cases with restriction of the atrial septum is greater in this series, at $53 \%$, than the previously reported rate of $22 \%,{ }^{4}$ although no details of the method of diagnosing this were given in this paper. This may simply be coincidental owing to the small number of cases in our series.

The hypoplastic left heart syndrome is detectable in the fetus by examination of the four chamber view and is one of the most common cardiac malformations detected in fetal life. ${ }^{1}$ The four chamber view is a standard view recommended by the ultrasound governing bodies as a routine part of an obstetric scan. Despite this, the condition is only detected prenatally in about $40 \%$ of cases seen as infants in the USA. ${ }^{13}$ As the results of staged palliative surgery have improved, more parents choose to continue with an affected pregnancy. It has become evident that the management of the neonate in the first days of life is crucial to the results of the first stage of the Norwood procedure..$^{14}$ Delivery of the mother close to the surgical centre and avoiding neonatal transfer appears to improve the results, ${ }^{23}$ but stabilisation with prostaglandins and balancing of the systemic and pulmonary resistances are also important. A restrictive atrial septal defect may contribute to haemodynamic instability in the first days of life. The ability to predict this complication prenatally may help in the immediate postnatal management of the affected infant. In addition, early surgical treatment can be scheduled.

The authors would like to thank Robert Siacca for the statistical analysis

1 Allan LD, Sharland GK, Milburn A, et al. Prospective diagnosis of 1,006 consecutive cases of congenital heart disease in the fetus. $\mathcal{F}$ Am Coll Cardiol 1994;23:1452-8.

2 Chang AC, Huhta JC, Yoon GY, et al. Diagnosis, transport and outcome in fetuses with left ventricular outflow obstruction. F Thorac Cardiovasc Surg 1991;102:841-6.

3 Tworetzky W, McElhinney DB, Reddy VM, et al. Does prenatal diagnosis of hypoplastic left heart syndrome lead to improved surgical outcome [abstract]. $7 \mathrm{Am}$ Coll Cardiol 1998;814(suppl):71A.

4 Forbess JM, Cook N, Roth SJ, et al. Ten-year institutional experience with palliative surgery for hypoplastic left heart syndrome. Risk factors related to stage I mortality. Circulation 1995;92(suppl):II-262-6.

5 Sahn DJ, Rice MJ, Silverman NH, et al. Poor prognosis for fetuses with restrictive foramen ovale and left heart obstructive lesions: a multicenter study [abstract]. $\mathcal{f} \mathrm{Am}$ Coll Cardiol 1996;27(suppl):158A

6 Keren G, Sherez J, Megidish R, et al. Pulmonary venous flow pattern-its relationship to cardiac dynamics. Circulation 1985;71:1105-12.

7 Klein AL, Tajik AJ. Doppler assessment of pulmonary venous flow in healthy subjects and in patients with heart disease. F Am Soc Echocardiogr 1991;4:379-92.

8 Basnight MA, Gonzalez MS, Kershenovich SC, et al. Pulmonary venous flow velocity : relation to hemodynamics, mitral flow velocity and left atrial volume and ejection fraction $\mathcal{F}$ Am Soc Echocardiogr 1991;4:547-58. 
9 Laudy JA, Huisman TW, de Ridder MA, et al. Normal fetal pulmonary venous blood flow velocity. Ultrasound Obstet pulmonary venous blood

10 Better DJ, Kaufman S, Allan LD. The normal pattern of pulmonary venous flow on pulsed Doppler examination of the human fetus. F Am Soc Echocardiogr 1996;9:281-5.

11 Sutton MS, Groves A, MacNeill A, et al. Assessment of changes in blood flow through the lungs and foramen ovale in the normal human fetus with gestational age: a prospective Doppler echocardiographic study. Br Heart $\mathcal{F}$ 1994;71: $232-7$
12 Rasanen J, Wood DC, Weiner S, et al. Role of the pulmonary circulation in the distribution of human fetal cardiac output during the second half of pregnancy. Circulation 1996;94:1068-73

13 Montana E, Khoury MJ, Cragan JD, et al. Trends and outcomes of prenatal diagnosis of congenital cardiac malformations by fetal echocardiography in a well defined birth population, Atlanta, Geor

14 Bove EL, Lloyd TR. Staged reconstruction for hypoplastic left heart syndrome. Ann Surg 1996;224:387-95. 